\title{
Spatial relational learning persists following neonatal hippocampal lesions in macaque monkeys
}

\author{
Pierre Lavenex $^{1,2}$, Pamela Banta Lavenex ${ }^{1,2}$ \& David G Amaral ${ }^{1}$
}

\begin{abstract}
The hippocampus is important for the acquisition of spatial representations of the environment and consequently in contextual memory. This suggests that the neural substrates underlying spatial cognition might be essential for remembering specific life episodes. Indeed, hippocampal lesions prevent spatial relational learning in adult rodents and monkeys, and result in profound amnesia in adult humans. In contrast, we show here that monkeys with neonatal hippocampal lesions learned new spatial relational information. Our experiments suggest that early hippocampal damage leads to functional brain reorganization that enables spatial information to be acquired through the use of brain regions that normally do not subserve this function.
\end{abstract}

The hippocampal formation, a group of cortical brain regions located in the medial temporal lobe, is essential for memory function. Behavioral and electrophysiological experiments in rodents have shown that hippocampal integrity is critical for learning and remembering a particular location in the absence of local cues: that is, for forming an allocentric (viewpoint-independent) representation of space that codes the goal location in relation to distant environmental cues $^{1-6}$. Recent experiments demonstrate that selective hippocampal damage prevents spatial relational learning in freely moving adult rhesus monkeys (Macaca mulatta) ${ }^{7}$. Studies of human hippocampal function also highlight its involvement with allocentric representations of space ${ }^{8-12}$.

Here, we tested the ability of juvenile monkeys that received hippocampal lesions shortly after birth to learn new spatial relational information. Freely moving monkeys were allowed to forage for a preferred food that was hidden beneath cups distributed in an openfield arena (Fig. 1). Six potentially baited cups were located in two arrays of 3 distinct locations among 18 possible locations. Each day, three locations on one of the arrays were baited. Food locations changed pseudo-randomly among arrays between days. The six potentially baited locations were fixed in relation to distant environmental cues. Monkeys were tested in two conditions. In the first, the local cue condition, colored cups identified the potentially baited locations, so that monkeys could rely on either a visual guidance strategy (egocentric coding) $)^{1-6}$ or spatial relational information (allocentric coding) ${ }^{1-6}$ to discriminate between the potentially baited and never-baited locations. In the second, the spatial relational condition, all cups were the same neutral color, so that the monkeys had to rely on an allocentric representation of the environment to discriminate between the potentially baited and never-baited locations. Multiple goals and four pseudo-randomly chosen entrance points prevented the monkeys from relying on an egocentric strategy to identify food locations ${ }^{7,13}$.
In contrast to monkeys with adult hippocampal lesions ${ }^{7}$, we show that monkeys with neonatal hippocampal lesions showed normal spatial relational learning and memory.

\section{RESULTS}

Hyperactivity is a common repercussion of bilateral hippocampal damage in rats ${ }^{14,15}$. Consistent with this, juvenile monkeys that received selective, bilateral neonatal lesions of the hippocampus were hyperactive compared with sham-operated control monkeys. During an acclimation phase (when no cups or food were present in the openfield arena), monkeys with hippocampal lesions locomoted more than controls (groups: $F_{1,12}=7.507, P=0.0179$; Fig. 2a). Monkeys with hippocampal lesions also opened more cups during testing under both the local cue and spatial relational conditions, indicating that they were more active than control monkeys (groups: $F_{1,24}=4.946$, $P=0.0358$; Fig. 2b).

We evaluated the effects of selective, neonatal hippocampal lesions on the monkeys' ability to find food in the open-field arena. First, monkeys foraged for food that was hidden at fixed locations marked by local cues. The hippocampal-lesioned and sham-operated monkeys were equally capable of discriminating between potentially baited marked locations and never-baited unmarked locations (groups: $F_{1,12}=0.002$, $P=0.9626$; locations: $F_{4,48}=158.087, P<0.0001$; interaction: $F_{4,48}=0.069, P=0.9911$; Fig. 3).

Next, the monkeys foraged for food that was hidden at fixed locations that were not marked by local cues. As expected, the shamoperated monkeys were able to discriminate between potentially baited and never-baited locations based on their relations with distant environmental cues (locations: $F_{4,20}=12.394, P<0.0001$; Fig. 4a). Notably, monkeys with neonatal bilateral hippocampal lesions also discriminated between potentially baited and never-baited locations in the absence of local cues (locations: $F_{4,28}=27.847, P<0.0001$;

${ }^{1}$ Department of Psychiatry and Behavioral Sciences, Center for Neuroscience, California National Primate Research Center, The M.I.N.D. Institute, University of California at Davis, 2805 50 th St., Sacramento, California 95817, USA. ²Department of Medicine, Unit of Physiology, University of Fribourg, Chemin du Musée 5, CH-1700 Fribourg, Switzerland. Correspondence should be addressed to P.L. (pierre.lavenex@unifr.ch). 


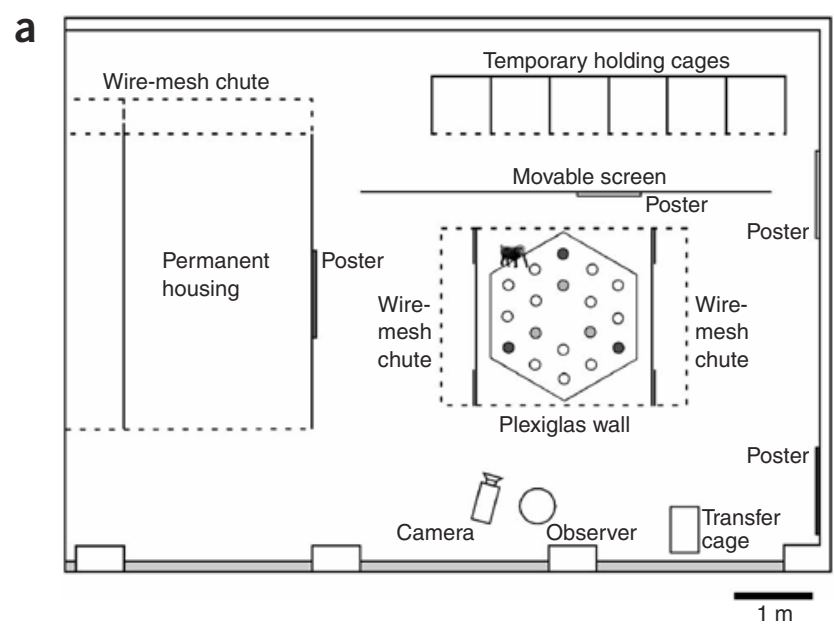

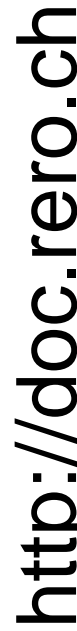

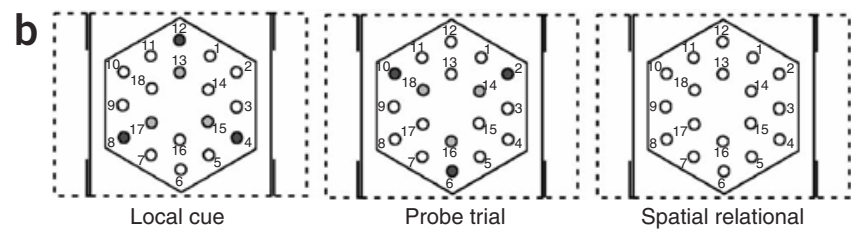

Fig. 4b). In fact, hippocampal-lesioned monkeys were as proficient as the control monkeys at discriminating between potentially baited and never-baited locations in the same array (groups: $F_{1,12}=4.005$, $P=0.0685$; locations: $F_{4,48}=34.123, P<0.0001$; interaction: $\left.F_{4,48}=1.1814, P=0.1415\right)$. The nearly significant group difference can be explained by differences in the levels of activity between the control and hippocampal-lesioned monkeys (Fig. 2). Because we normalized the number of choices in each category according to the probability of making that choice (Pot in/3, Pot out/3, Equ in/3, Equ out/3, Other/6), the differences between groups in the number of 'Other' cups opened are reflected in the overall group analysis.

Finally, a probe trial, in which the locations of the colored cups were shifted $60^{\circ}$ from their usual spatial locations (Fig. 1b), revealed that sham-operated and hippocampal-lesioned monkeys were equally reliant on local cues and spatial relational information to discriminate between potentially baited and never-baited locations when local cues were present (groups: $F_{1,8}=0.0002, P=0.9888$; locations: $F_{4,32}=$ 22.825, $P<0.0001$; interaction, $F_{4,32}=0.846, P=0.5064$; Fig. 5).

An evaluation of individual behavioral strategies showed that every juvenile monkey that received bilateral neonatal hippocampal lesions $(n=8)$ discriminated between the potentially baited and the neverbaited locations in the absence of local cues marking these locations (Supplementary Table 1 online). Thus, although juvenile monkeys with bilateral neonatal lesions of the hippocampus showed hyperactivity as compared to sham-operated, age-matched control monkeys, they still discriminated between unmarked potentially baited and neverbaited locations on the basis of their spatial relations with distant environmental cues.

We used magnetic resonance imaging analyses to characterize the extents of the hippocampal lesions (Fig. 6), as the monkeys that participated in this study are still undergoing behavioral testing in studies on the development of primate social behavior. The extent of the lesions was very consistent across all eight of the monkeys (Supplementary Figs. 1 and 2 online). The entorhinal and perirhinal cortices were largely, if not completely, intact in all of the lesioned monkeys. The intended targets, including the dentate gyrus, areas CA3,
Figure 1 Schematic representation of the testing environment and experimental conditions. (a) Aerial view of the experimental room. Eighteen plastic cups were regularly distributed on a hexagonal board $(210 \mathrm{~cm}$ in diameter), which was placed in a square testing arena $(220 \mathrm{~cm} \times 220 \mathrm{~cm} \times$ $220 \mathrm{~cm}$ ). The front panel, the roof and the top half of the back panel (dashed lines) were made of Plexiglas, allowing a clear view of distant environmental cues; two metal side panels (solid lines) provided visual barriers between the open-field arena and the wire-mesh holding chutes. Remotely operated sliding doors at each corner of the arena (double solid lines) allowed the monkeys to go in and out of the arena from wire-mesh chutes that were located along both sides. (b) Schematic representation of the arena in the different testing conditions. (1) For the local cue condition, blue cups marked potentially baited locations 4,8 and 12 on the outer array and red cups marked potentially baited locations 13,15 and 17 on the inner array. All of the other locations were covered with neutral (beige) cups. (2) For the probe trial, colored cups were shifted $60^{\circ}$ from the correct spatial locations. Blue cups were at locations 2, 6 and 10 and red cups were at locations 14, 16 and 18. Neutral cups were at locations 4, 8 and 12, and 13, 15 and 17, and all of the remaining locations. No food was present. (3) For the spatial relational condition, neutral cups covered the potentially baited locations 4 , 8 and 12 , and 13,15 and 17 , as well as all of the other locations.

CA2 and CA1, the subiculum, the presubiculum and the parasubiculum, were substantially damaged, if not completely eliminated bilaterally, in each monkey.

\section{DISCUSSION}

We have shown previously that selective hippocampal damage prevents allocentric spatial relational learning in freely moving adult monkeys ${ }^{7}$. Here we show that learning and retention of new allocentric spatial relational information were unimpaired in monkeys that received bilateral hippocampal lesions shortly after birth. The differential effect of early versus late hippocampal lesions on spatial relational learning in monkeys (Fig. 7) is reminiscent of the differential effect of early versus late hippocampal lesions on semantic learning in humans. Hippocampal lesions in adult human subjects impair both semantic and episodic memory ${ }^{16,17}$, whereas individuals with early hippocampal damage have deficits in episodic memory, but not in semantic memory ${ }^{18-20}$. Below, we discuss how the present results further our understanding of the relations between spatial memory, and semantic and episodic memory, as well as how functional brain reorganization might enable the acquisition of spatial memory following early, but not late, hippocampal lesions.

Spatial memory has often been considered in the context of episodic memory ${ }^{1,5,21,22}$. The importance of the hippocampus in the
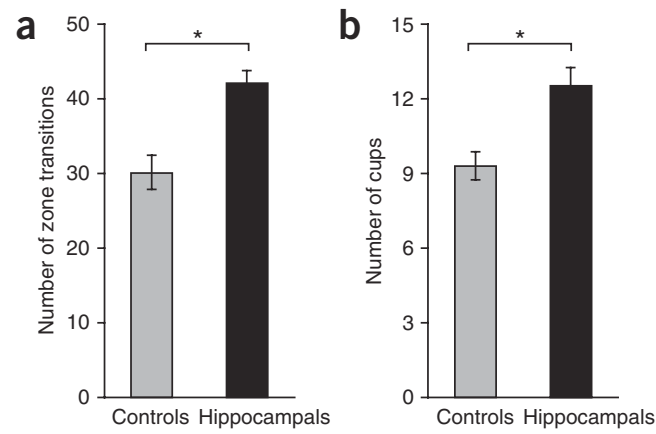

Figure 2 Indices of the overall activity level for sham-operated control and hippocampus-lesioned monkeys. (a) Number of zone transitions per session during 5-min acclimation sessions. (b) Number of cups opened per trial across the local cue and spatial relational conditions. Data are given as mean \pm s.e. $m$. 

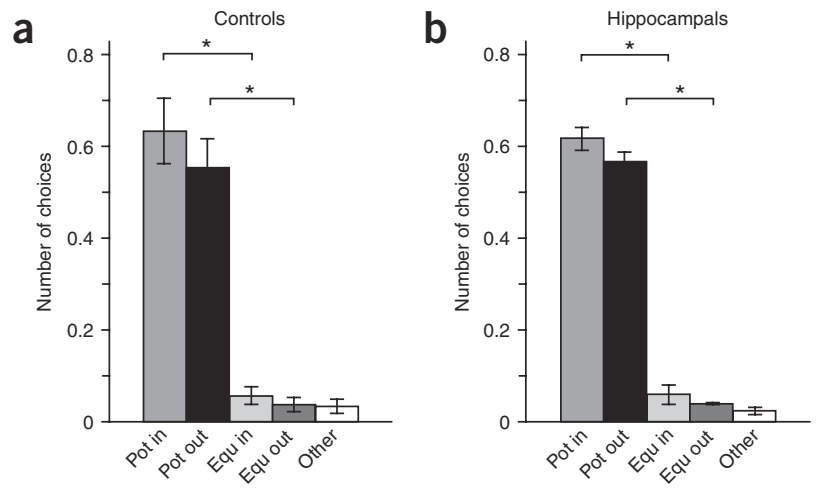

Figure 3 Monkeys' first four choices during standard trials in the local cue condition. (a) Sham-operated, control monkeys. (b) Hippocampal-lesioned monkeys. The choices were 'Pot in', potentially baited locations at the corners of the inner hexagon (locations 13, 15 and 17); 'Pot out', potentially baited locations at the corners of the outer hexagon (locations 4, 8 and 12); 'Equ in', never-baited locations at the corners of the inner hexagon (locations 14,16 and 18), termed 'equivalent' because of their position at one of the three corners of the hexagon, topologically equivalent to the position of the potentially baited locations; 'Equ out', never-baited locations at the corners of the outer hexagon (locations 2, 6 and 10); and 'Other', never-baited locations on the sides of the outer hexagon (locations 1, 3, 5, 7, 9 and 11). The number of choices in each category $(n)$ is normalized according to the probability of making that choice. For both groups, Pot in $=$ Pot out $>$ Equ in $=$ Equ out $=$ Other, all $P<0.0001$. Data are given as mean \pm s.e.m.

processing of allocentric spatial representations, and consequently in contextual memory, suggests that the neural substrates underlying spatial cognition might be essential for recalling specific life episodes ${ }^{12,22}$. According to the cognitive map theory of hippocampal function ${ }^{3,5}$, the hippocampus is essential for acquisition, maintenance and retrieval of all aspects of the allocentric (spatial relational) coding of space. Recent experiments, however, have reported that profoundly amnesic patients ${ }^{23,24}$, as well as animals with experimental lesions including complete destruction of the hippocampus ${ }^{25}$, show preserved spatial memory for locations that were learned long before the hippocampal damage occurred (remote spatial memory). This is similar to the way that amnesic individuals show preserved episodic and semantic memory for information that was learned long before hippocampal damage ${ }^{26}$. These findings have led some researchers to re-evaluate the function of the hippocampal formation in spatial memory, suggesting that the hippocampus is critical for the acquisition of new allocentric representations of the environment ${ }^{12,21,22}$, but not for their long-term maintenance or retrieval ${ }^{23-25}$. Our previous studies of monkeys with adult hippocampal lesions support this view ${ }^{7}$, but our current findings do not, as

Figure 5 Monkeys' choices (all cups that were opened) in the dissociation probe trial (no food present). (a) Sham-operated control monkeys:

Color in $=$ Color out $=$ Space in $=$ Space out $>$ Other; all $P<0.0001$. (b) Hippocampal-lesioned monkeys: Color in $=$ Color out $=$ Space out $>$ Other; Color in = Space out $>$ Space In $>$ Other; all $P<0.02$. The choices were 'Color in', red cups at never-baited locations at the corners of the inner hexagon (locations 14, 16 and 18); 'Color out', blue cups at never-baited locations at the corners of the outer hexagon (locations 2, 6 and 10); 'Space in', neutral cups at correct spatial locations at the corners of the inner hexagon (locations 13, 15 and 17); 'Space out', neutral cups at correct spatial locations at the corners of the outer hexagon (locations 4, 8 and 10); and 'Other', neutral cups at never-baited locations on the sides of the outer hexagon (locations 1, 3, 5, 7, 9 and 11). Data are given as mean \pm s.e.m.
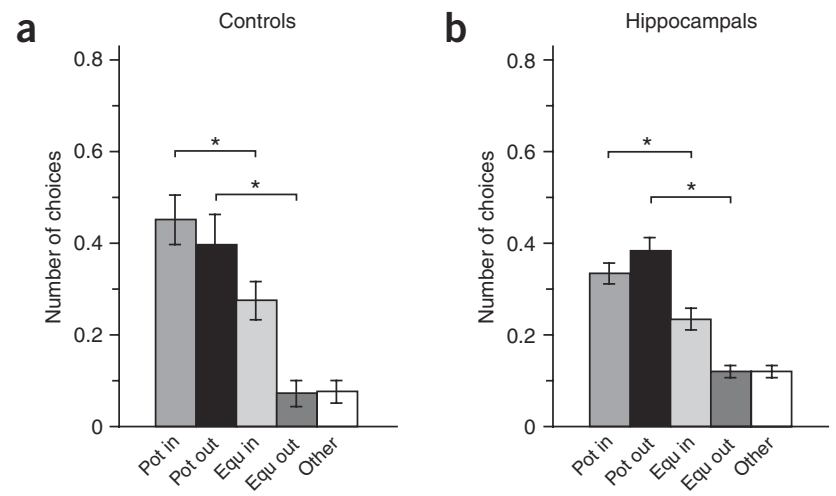

Figure 4 Monkeys' first four choices during standard trials in the spatial relational condition. (a) Sham-operated control monkeys: Pot in $>$ Equ in $>$ Equ out $=$ Other, all $P<0.023$; Pot out $>$ Equ out $=$ Other, $P=0.0002$. (b) Hippocampal-lesioned monkeys: Pot in $=$ Pot out $>$ Equ in $>$ Equ out $=$ Other; all $P<0.007$. Data are given as mean \pm s.e.m. For abbreviations, see Figure $\mathbf{3}$.

we observed that spatial relational learning persisted after neonatal hippocampal lesions.

Although spatial memories have rarely been considered in the context of semantic memory, a functional and neuroanatomical distinction was recently proposed between detailed, episode-specific spatial representations of experienced environments (analogous to autobiographical memory) and schematic, episode-free representations of the topography of the environment (analogous to semantic memory $)^{22,27}$. Our current findings show that episode-free, spatial relational learning was preserved in monkeys that received bilateral hippocampal lesions shortly after birth, whereas hippocampal lesions in adults prevent spatial relational learning ${ }^{7}$. Our results parallel those showing that humans with early hippocampal damage show episode-free semantic learning ${ }^{18-20,28}$, whereas hippocampal lesions in adults impair semantic learning ${ }^{16,17}$. In seeming contrast with our findings, however, anecdotal observations by family members and virtual reality studies indicate impaired allocentric processing in humans with early hippocampal damage $\mathrm{e}^{19-21,29-31}$. It is possible, however, that the anecdotal observations reported in human individuals reflect episode-specific spatial memory impairments ${ }^{22}$. For example, remembering where objects and belongings are located on a daily basis requires the ability to integrate specific temporal information, which is fundamental to episodic memory and is sensitive to hippocampal damage $e^{5,21,22,32-34}$. With respect to virtual reality studies, the dynamic process of extracting spatial information
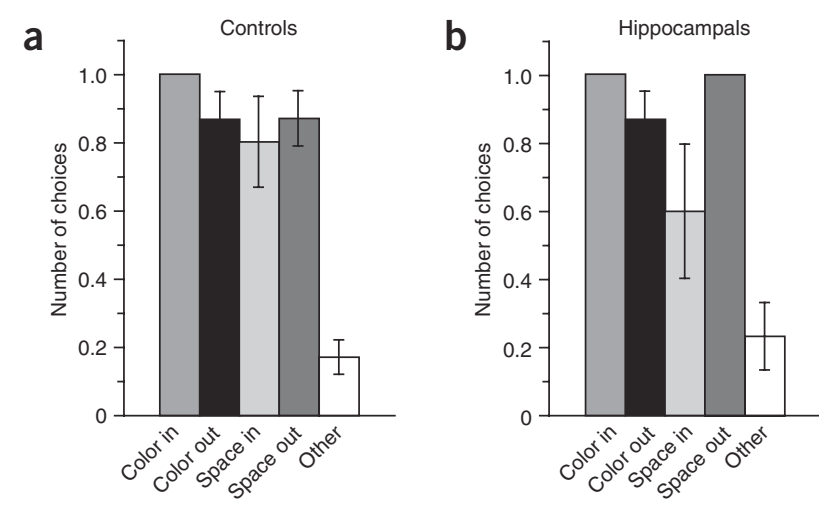
a

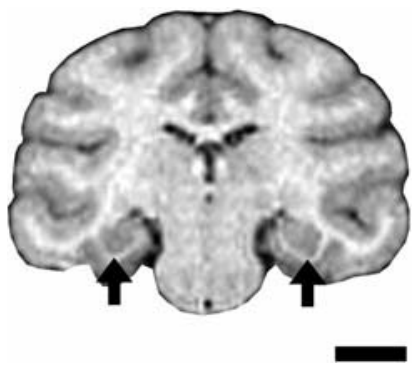

d

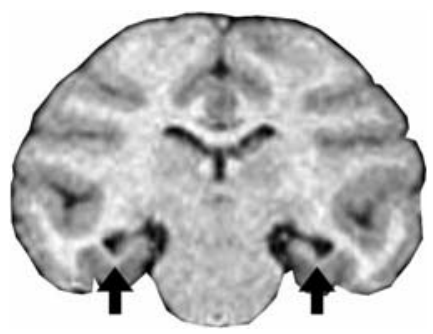

b

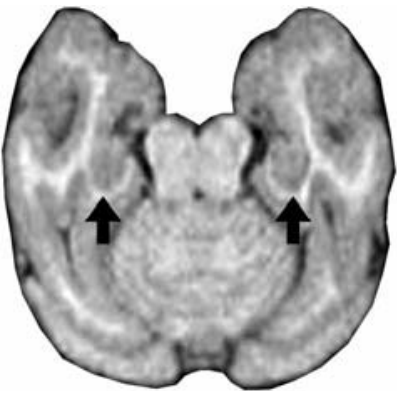

e

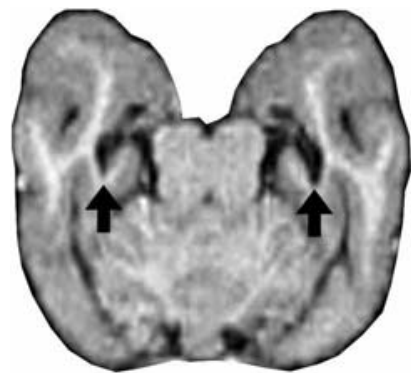

C

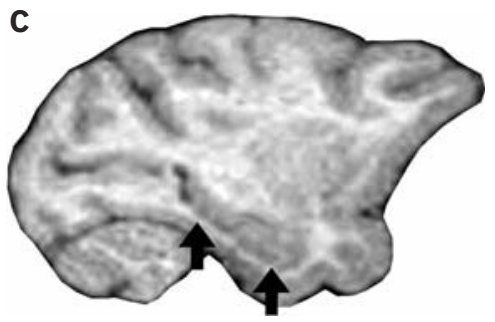

f

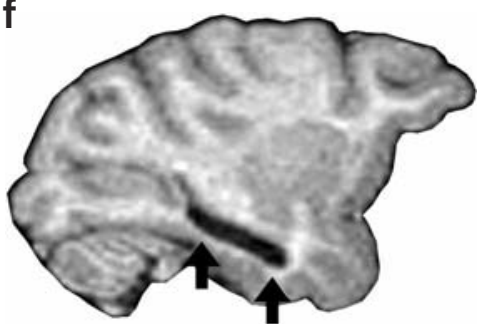

Figure 6 Magnetic resonance images showing the medial temporal lobe region in three different section planes (coronal, horizontal and sagittal). (a-c) Sham-operated control monkey (MMU33218). (d-f) Representative experimentally lesioned monkey (MMU33258). Arrows indicate the hippocampus in the control monkey, shown in a-c, and the corresponding damaged area in the experimentally lesioned monkey, shown in $\mathbf{d}-\mathbf{f}$. The scale bar in $\mathbf{a}$ is $1 \mathrm{~cm}$ and applies to all panels.

in a virtual reality task might differ from that performed while a subject is freely and actively navigating in a real environment ${ }^{21}$. In virtual reality experiments, deficits in allocentric processing are typically demonstrated by impaired memory for object locations when the viewpoint is shifted between presentation and retrieval, as compared with that observed when the viewpoint remains the same $e^{21,29-31}$. The finding that reaction times in normal individuals increase as the angle between the viewpoints increases suggests that the allocentric system responsible for solving this shifted-view task depends on the manipulation of viewpoint-dependent representations using egocentric information encoded at presentation ${ }^{29,30}$. In contrast, when a subject is actively navigating in the real world, self-motion information might enable automatic updating of the representation of object locations in relation to distant environmental cues that are perceived from contiguous viewpoints. Object locations could therefore be directly encoded into a seamless allocentric representation of the environment ${ }^{5,21}$. Our findings indicate that such processes can be sustained by extra-hippocampal brain areas after early hippocampal damage, but not in subjects who suffer hippocampal damage later in life.

\footnotetext{
Figure 7 Differential effect of early versus late hippocampal lesions. The first four choices made during standard trials in the spatial relational condition show the differential effect of early versus late hippocampal lesions on spatial relational learning (groups: $F_{1,12}=0.001, P=0.9722$; locations: $F_{4,48}=42.045, P<0.0001$; interaction: $F_{4,48}=17.712, P<0.0001$ ). Juvenile monkeys with bilateral neonatal hippocampal lesions (a) were able to discriminate between potentially baited and never-baited locations (locations: $F_{4,28}=27.847, P<0.0001$; Pot in $=$ Pot out $>$ Equ in $>$ Equ out $=$ Other; all $P<0.007$ ), whereas monkeys that received bilateral hippocampal lesions in adulthood (b) were incapable of discriminating between potentially baited and never-baited locations (data from ref. 7) (locations: $F_{4,20}=32.187, P<0.0001$; Pot in $=$ Equ in $>$ all others, all $P<0.0001 ;$ Pot out $=$ Equ out $=0$ Other; all $P>0.31)$. Data are given as mean \pm s.e.m. For abbreviations, see Figure 3 .
}

The rearrangement of synaptic connections is potentially the most important biological mechanism involved in the recovery of function after brain injury ${ }^{35-37}$. Mammals, including humans, show a remarkable recovery of behavioral functions after circumscribed brain injuries, particularly those occurring early in life ${ }^{38-40}$. Accordingly, some have suggested that the functional recovery that enables semantic learning in humans is contingent on early injury to the hippocampal formation, allowing for a compensatory neural reorganization ${ }^{19,20,28}$. Similarly, we propose that the extra-hippocampal cortical regions, which are specialized for the maintenance and retrieval of long-term, episode-free spatial memories, undergo a functional reorganization to enable the acquisition of these memories following early, but not late, hippocampal damage. The potential morphological reorganization of these brain regions following early hippocampal lesions will be the subject of future studies in this research program.

\section{METHODS}

Experimental subjects. The subjects were 16 infant macaque monkeys (Macaca mulatta) born and raised at the California National Primate Research Center. Control and hippocampal-lesion surgeries were performed 12-16 d after birth,
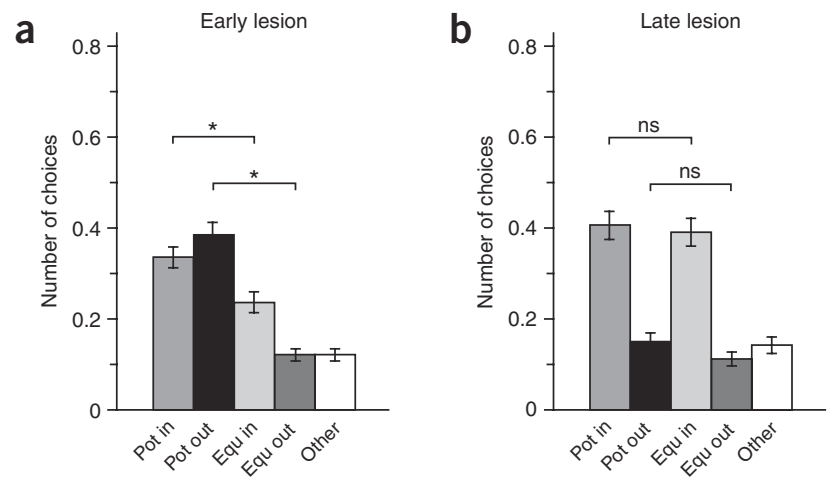
following previously described protocols ${ }^{41}$. Infants were reared by their mothers in a socialization cohort consisting of six mother-infant pairs and one adult male that met for a minimum of $3 \mathrm{~h}$ per d, $5 \mathrm{~d}$ per week in a large cage. Infants were weaned from their mothers when the youngest member of each cohort reached 6 months of age. From then onwards, infants were permanently housed with their previously established cohort of six infants, one adult male and a new adult female ${ }^{41}$. These experiments were approved by the Institutional Animal Care and Use Committee of the University of California at Davis and were in accordance with the US National Institute of Health guidelines for the use of animals in research. Monkeys were not subjected to any food or water restrictions, except that they did not receive their regular morning rations until after the daily testing session was completed. Monkeys were tested at the same time each day ( $5 \mathrm{~d}$ per week), between 7:30 and 11:00 a.m.

Pre-training. Monkeys began pre-training and acclimation $18.6 \pm 0.3$ months after surgery (sham-operated: $18.9 \pm 0.6$ months; hippocampus-lesioned: $18.5 \pm 0.4$ months). During a pre-training phase that took place in temporary holding cages $(61 \mathrm{~cm} \times 66 \mathrm{~cm} \times 81 \mathrm{~cm})$, monkeys were first trained to displace a plastic cup to retrieve grapes that were hidden underneath $i^{7,13}$. Following pre-training, monkeys received one 5-min acclimation session per day for $5 \mathrm{~d}$, during which they were free to explore the open-field arena. During this phase, no cups or food were present in the arena.

Open-field testing. Monkeys foraged freely for grapes that were hidden beneath cups in two different arrays of 3 distinct locations among 18 locations that were distributed in the open-field arena (Fig. 1). Monkeys were given 3 trials per $\mathrm{d}$ (with a 1-min inter-trial interval), $5 \mathrm{~d}$ per week. Each day, three locations on either the inner array or the outer array were baited. The location of the food changed pseudo-randomly between the two arrays between days, but remained the same between trials within a daily session ${ }^{7,13}$. For each trial, monkeys entered and exited the arena from one of the four pseudo-randomly chosen entrances. The orientation of the entire apparatus remained fixed in relation to distant environmental cues within the testing room. The board on which the cups were distributed, however, was rotated clockwise $60^{\circ}$ before each trial to make any uncontrolled local cues irrelevant, but the grapes (and local cues if present) were always placed in exactly the same locations in relation to distant environmental cues. Monkeys were tested over an 8-week period and experienced two different, alternating testing conditions: a local cue and a spatial relational condition.

In the local cue condition, monkeys were tested for their ability to find food at fixed locations that were marked by colored cups (Fig. 1b). Although the particular array that was baited changed pseudo-randomly between days, both sets of local cues were present on the board during all local cue sessions. We considered 2 weeks of experience ( $5 \mathrm{~d}$ per week) with 3 trials per $\mathrm{d}$ ( $10 \mathrm{~d}$ or 30 trials) in the local cue condition for data analysis. The analysis of the first four cups that were opened in the first phase of testing in the local cue condition did not reveal any group difference, indicating that task acquisition was similar between the lesion conditions (data not shown).

In the spatial relational condition, no local cues marked the potentially baited locations (Fig. 1b). Therefore, monkeys could not discriminate between potentially baited and never-baited locations based on local features. Furthermore, the combination of multiple goal locations (six) and four pseudorandomly chosen entrance points precluded the ability of monkeys to rely on an egocentric strategy to identify food locations. We considered 2 weeks of experience ( $5 \mathrm{~d}$ per week) with 3 trials per $\mathrm{d}$ ( $10 \mathrm{~d}$ or 30 trials) in the spatial relational condition for data analysis.

In addition, on one trial in the local cue condition, the colored cups were shifted $60^{\circ}$ from their usual spatial locations and no food was present (Fig. 1b). During this dissociation probe trial, monkeys encountered the same pattern of cups distributed throughout the arena, but the absolute locations of the colored cups were shifted in relation to distant environmental cues, thus rendering the local and spatial relational information incoherent.

Data analysis. Movement activity during acclimation was determined by recording the position of the monkey among eight zones subdividing the open-field arena every $5 \mathrm{~s}$ for the duration of each 5 -min session. Activity level was also assessed by analyzing the number of cups that the monkeys opened during each standard trial in the local cue and spatial relational conditions.
Statistical analyses were conducted using analyses of variance (ANOVAs), with lesion condition as a factor and acclimation sessions or testing conditions as the repeated measures. The assessment of normality was performed graphically with a $z$-score histogram.

To identify the strategies that control and hippocampal-lesioned monkeys used to discriminate between the baited and the non-baited locations in the local cue and spatial relational conditions, we classified each of the 18 locations into one of five categories, with respect to whether it could be baited and what its position was in the open-field arena. For each analysis, we normalized the number of choices of a particular type based on the probability of making that choice. For standard trials, the numbers of choices of 'Pot in', 'Pot out', 'Equ in' and 'Equ out' were divided by 3 and the number of 'Other' choices was divided by 6 . For the probe trial, the numbers of choices of 'Color in', 'Color out', 'Space in' and 'Space out' were divided by 3 and the number of 'Other' choices (the never-baited locations on the sides of the outer hexagon: locations $1,3,5,7,9,11$ ) was divided by 6 . We report the first four choices for the local cue and spatial relational trials, and all choices made during the dissociation probe trial.

Statistical analyses were conducted using ANOVAs, with lesion condition as a factor and choice type (locations) as the repeated measures. Post-hoc analyses were carried out with Fisher-PLSD tests. Within-group analyses were conducted with choice type as the repeated measures. Because monkeys might have unique strategies for finding the food locations ${ }^{13}$, we also analyzed the behavior of each individual monkey separately to detect evidence of learning. In this case, statistical analyses were performed within subjects, with choice type as a factor and daily sessions as the repeated measures. Statview 5.0.1 statistical software was used to perform all statistical analyses (SAS Institute Inc.).

Lesion evaluation. Lesion size and placement were analyzed by magnetic resonance images. At $50.0 \pm 0.3$ months of age, control and hippocampuslesioned monkeys were imaged using a previously described imaging para$\operatorname{digm}^{41}$. MRI images were analyzed using the OsiriX open source imaging software ${ }^{42}$. Briefly, DICOM MRI images were uploaded and a two-dimensional multi-planar reconstruction (2D MPR) was generated to perform a detailed evaluation of the extent of the lesion. The nomenclature and boundaries of the different structures of the medial temporal lobe followed our previously published descriptions ${ }^{43-45}$.

Note: Supplementary information is available on the Nature Neuroscience website.

\section{ACKNOWLEDGMENTS}

We thank J. Bennett and P. Tennant for assistance with surgeries and J. Toscano and J. Burky for assistance with behavioral testing. This research was conducted at the California National Primate Research Center (NIH, base grant RR00169) and was supported by grants from the US National Institute of Health (RO1NS16980, RO1-MH57502), a Faculty and Alumni Research Development Fund grant from the Department of Psychiatry and Behavioral Sciences at the University of California at Davis and a grant from the Swiss National Science Foundation (PP00A-106701).

\section{COMPETING INTERESTS STATEMENT}

The authors declare that they have no competing financial interests.

Published online at http://www.nature.com/natureneuroscience

Reprints and permissions information is available online at http://npg.nature.com/ reprintsandpermissions

1. Eichenbaum, $\mathrm{H}$. The hippocampus, memory, and place cells: is it spatial memory or a memory space? Neuron 23, 209-226 (1999).

2. Morris, R.G., Garrud, P., Rawlins, J.N.P. \& Okeefe, J. Place navigation is impaired in rats with hippocampal-lesions. Nature 297, 681-683 (1982).

3. Nadel, L. The hippocampus and space revisited. Hippocampus 1, 221-229 (1991).

4. O'Keefe, J. \& Dostrovsky, J. The hippocampus as a spatial map. Preliminary evidence from unit activity in the freely-moving rat. Brain Res. 34, 171-175 (1971).

5. O'Keefe, J. \& Nadel, L. The Hippocampus as a Cognitive Map (Clarendon Press, Oxford, 1978)

6. Schenk, F., Grobéty, M.-C., Lavenex, P. \& Lipp, H.-P. Dissociation between basic components of spatial memory in rats. in Behavioural Brain Research in Naturalistic and Semi-Naturalistic Settings NATO ASI series, Series D, Behavioural and Social Sciences (eds. Alleva, E., Fasolo, A., Lipp, H.-P., Nadel, L. \& Ricceri, L.) 277-300 (Kluwer, Dordrecht, The Netherlands, 1995). 
7. Banta Lavenex, P., Amaral, D.G. \& Lavenex, P. Hippocampal lesion prevents spatial relational learning in adult macaque monkeys. J. Neurosci. 26, 4546-4558 (2006).

8. Astur, R.S., Taylor, L.B., Mamelak, A.N., Philpott, L. \& Sutherland, R.J. Humans with hippocampus damage display severe spatial memory impairments in a virtual Morris water task. Behav. Brain Res. 132, 77-84 (2002).

9. Bohbot, V.D., Iaria, G. \& Petrides, M. Hippocampal function and spatial memory: evidence from functional neuroimaging in healthy participants and performance of patients with medial temporal lobe resections. Neuropsychology 18, 418-425 (2004).

10. Parslow, D.M. et al. Allocentric spatial memory activation of the hippocampal formation measured with fMRI. Neuropsychology 18, 450-461 (2004).

11. Shelton, A.L. \& Gabrieli, J.D.E. Neural correlates of individual differences in spatial learning strategies. Neuropsychology 18, 442-449 (2004).

12. Nadel, L. \& Hardt, O. The spatial brain. Neuropsychology 18, 473-476 (2004).

13. Lavenex, P. \& Banta Lavenex, P. Spatial relational memory in 9-month-old macaque monkeys. Learn. Mem. 13, 84-96 (2006).

14. Coutureau, E., Galani, R., Jarrard, L.E. \& Cassel, J.C. Selective lesions of the entorhinal cortex, the hippocampus, or the fimbria-fornix in rats: a comparison of effects on spontaneous and amphetamine-induced locomotion. Exp. Brain Res. 131, 381-392 (2000).

15. Whishaw, I.Q. \& Jarrard, L.E. Similarities vs. differences in place learning and circadian activity in rats after fimbria-fornix section or ibotenate removal of hippocampal cells. Hippocampus 5, 595-604 (1995).

16. Rempel-Clower, N.L., Zola, S.M., Squire, L.R. \& Amaral, D.G. Three cases of enduring memory impairment after bilateral damage limited to the hippocampal formation. J. Neurosci. 16, 5233-5255 (1996).

17. Zola-Morgan, S., Squire, L.R. \& Amaral, D.G. Human amnesia and the medial temporal region enduring memory impairment following a bilateral lesion limited to field CA1 of the hippocampus. J. Neurosci. 6, 2950-2967 (1986).

18. Baddeley, A., Vargha-Khadem, F. \& Mishkin, M. Preserved recognition in a case of developmental amnesia: implications for the acquisition of semantic memory? J. Cogn. Neurosci. 13, 357-369 (2001).

19. Gadian, D.G. et al. Developmental amnesia associated with early hypoxic-ischaemic injury. Brain 123, 499-507 (2000).

20. Vargha-Khadem, F. et al. Differential effects of early hippocampal pathology on episodic and semantic memory. Science 277, 376-380 (1997).

21. Burgess, N., Maguire, E.A. \& O'Keefe, J. The human hippocampus and spatial and episodic memory. Neuron 35, 625-641 (2002).

22. Moscovitch, M. et al. Functional neuroanatomy of remote episodic, semantic and spatial memory: a unified account based on multiple trace theory. J. Anat. 207, 35-66 (2005).

23. Rosenbaum, R.S. et al. Remote spatial memory in an amnesic person with extensive bilateral hippocampal lesions. Nat. Neurosci. 3, 1044-1048 (2000).

24. Teng, E. \& Squire, L.R. Memory for places learned long ago is intact after hippocampal damage. Nature 400, 675-677 (1999).

25. Winocur, G., Moscovitch, M., Caruana, D.A. \& Binns, M.A. Retrograde amnesia in rats with lesions to the hippocampus on a test of spatial memory. Neuropsychologia $\mathbf{4 3}$, 1580-1590 (2005).

26. Milner, B., Squire, L.R. \& Kandel, E.R. Cognitive neuroscience and the study of memory. Neuron 20, 445-468 (1998).
27. Moscovitch, M., Nadel, L., Winocur, G., Gilboa, A. \& Rosenbaum, R.S. The cognitive neuroscience of remote episodic, semantic and spatial memory. Curr. Opin. Neurobiol. 16, 179-190 (2006).

28. Duzel, E., Vargha-Khadem, F., Heinze, H.J. \& Mishkin, M. Brain activity evidence for recognition without recollection after early hippocampal damage. Proc. Natl. Acad. Sci. USA 98, 8101-8106 (2001).

29. King, J.A., Burgess, N., Hartley, T., Vargha-Khadem, F. \& O'Keefe, J. Human hippocampus and viewpoint dependence in spatial memory. Hippocampus 12, 811-820 (2002).

30. King, J.A., Trinkler, I., Hartley, T., Vargha-Khadem, F. \& Burgess, N. The hippocampal role in spatial memory and the familiarity-recollection distinction: A case study. Neuropsychology 18, 405-417 (2004).

31. Spiers, H.J., Burgess, N., Hartley, T., Vargha-Khadem, F. \& O'Keefe, J. Bilatera hippocampal pathology impairs topographical and episodic memory but not visual pattern matching. Hippocampus 11, 715-725 (2001).

32. Eichenbaum, $\mathrm{H}$. The hippocampus, memory, and place cells: is it spatial memory of a memory space? Neuron 23, 209-226 (1999).

33. Squire, L.R., Stark, C.E. \& Clark, R.E. The medial temporal lobe. Annu. Rev. Neurosci. 27, 279-306 (2004).

34. Tulving, E. \& Markowitsch, H.J. Episodic and declarative memory: Role of the hippocampus. Hippocampus 8, 198-204 (1998).

35. Deller, T. \& Frotscher, M. Lesion-induced plasticity of central neurons-sprouting of single fibres in the rat hippocampus after unilateral entorhinal cortex lesion. Prog. Neurobiol. 53, 687-727 (1997).

36. Goldman, P.S. Neuronal plasticity in primate telencephalon anomalous projections induced by prenatal removal of frontal cortex. Science 202, 768-770 (1978).

37. Steward, 0. Lesion-induced synapse reorganization in the hippocampus of cats: sprouting of entorhinal, commisural/associational, and mossy fiber projections after unilateral entorhinal cortex lesions, with comments on the normal organization of these pathways. Hippocampus 2, 247-268 (1992).

38. Goldman, P.S. \& Galkin, T.W. Prenatal removal of frontal association cortex in the fetal rhesus monkey anatomical and functional consequences in postnatal life. Brain Res. 152, 451-486 (1978).

39. Neville, H.J. The development and neural bases of higher cognitive functions. Ann. NY Acad. Sci. 608, 71-87 (1990).

40. Villablanca, J.R. \& Hovda, D.A. Developmental neuroplasticity in a model of cerebra hemispherectomy and stroke. Neuroscience 95, 625-637 (2000).

41. Bauman, M.D., Lavenex, P., Mason, W.A., Capitanio, J.P. \& Amaral, D.G. The development of mother-infant interactions after neonatal amygdala lesions in rhesus monkeys. J. Neurosci. 24, 711-721 (2004).

42. Rosset, A., Spadola, L. \& Ratib, O. OsiriX: an open-source software for navigating in multidimensional DICOM images. J. Digit. Imaging 17, 205-216 (2004).

43. Amaral, D.G. \& Lavenex, P. Hippocampal neuroanatomy. in The Hippocampus Book (eds. Andersen, P., Morris, R.G.M., Amaral, D.G., Bliss, T. \& O'Keefe, J.) 37-114 (Oxford University Press, Oxford, UK, 2006).

44. Lavenex, P. \& Amaral, D.G. Hippocampal-neocortical interaction: a hierarchy of associativity. Hippocampus 10, 420-430 (2000).

45. Schumann, C.M. et al. The amygdala is enlarged in children, but not adolescents, with autism; the hippocampus is enlarged at all ages. J. Neurosci. 24, 6392-6401 (2004). 